\title{
On the solvability conditions of the first boundary value problem for a system of elliptic equations that strongly degenerate at a point
}

\author{
Stasys Rutkauskas
}

\section{Correspondence: stasys. rutkauskas@mii.vu.It \\ Institute of Mathematics and Informatics of Vilnius University, Akademijos str. 4, LT-08863, Vilnius, Lithuania}

\begin{abstract}
A system of elliptic equations which are irregularly degenerate at an inner point is considered in this article. The equations are weakly coupled by a matrix that has multiple zero eigenvalue and corresponding to it adjoint vectors. Two statements of a well-posed Dirichlet type problem in the class of smooth functions are given and sufficient conditions on the existence and uniqueness of the solutions are obtained.

Keywords: systems of elliptic equations, degenerate elliptic equations, boundary value problems, Dirichlet type problem
\end{abstract}

\section{Introduction and statement of the problems}

The first results in the area of boundary value problems for an elliptic equation with degeneracy at an inner point of the considered domain are obtained in [1]. In that study, the Dirichlet problem for a weakly (regularly) degenerating elliptic equation with the main part of Laplace's operator is studied. These results are developed in [2], where the degenerate elliptic operator is generalized and, over and above, the second boundary value problem is investigated. In [3], the existence of a weak solution to the Dirichlet problem for an elliptic equation degenerating at isolated points in the class of Hölder functions is proved. In the case of the strong (irregular) degeneracy, can new effects emerge which influence the well-posedness of the boundary value problems. For instance, in [4], it is shown that in a well-posed Dirichlet type problem the asymptotic of the solution near the degeneracy point is supposed to be known. Many more difficulties come into being in the investigation of the systems of degenerate elliptic equations. Some results for weakly related degenerate elliptic systems are obtained in [5-7]. Particularly, these articles deal with Dirichlet type problems for the elliptic system

$$
a(r) \Delta u+\sum_{i=1}^{n} B_{i}(x) u_{x_{i}}+C(x) u=0, \quad x \in D,
$$

where $r=|x|, a$ is a continuous function such that $a(r)=o(1)$ as $r \rightarrow 0$, and $a(r)>0$ for $r>0, x=0$ is an inner point of domain $D, \Delta$ is Laplace's operator, $B_{i}(x)$ and $C(x)$ are diagonal and square matrices, consequently, which are smooth enough in $\bar{D}$. In $[5,6]$, the Dirichlet problem in the class of vector functions $u$ bounded in $D_{0}=D \backslash\{x=0\}$ is

(c) 2011 Rutkauskas; licensee Springer. This is an Open Access article distributed under the terms of the Creative Commons Attribution License (http://creativecommons.org/licenses/by/2.0), which permits unrestricted use, distribution, and reproduction in any medium, provided the original work is properly cited. 
solved under the assumption that elements of the matrices $B_{i}(x)$ tend to zero, as $x \rightarrow 0$, fast enough. In [7], a weighted Dirichlet problem with supplementary weighted condition of the shape

$$
\lim _{x \rightarrow 0}\left(\Psi(x) u(x)-h\left(\frac{x}{r}\right)\right)=0
$$

is considered under the condition $a(r)=O\left(r^{2 \alpha}\right), \alpha>1$, as $r \rightarrow 0$. In the same study, $\Psi$ $(x)$ is some matrix entries of which are decreasing as $x \rightarrow 0$, and $h$ is a given vector function smooth on the unit sphere. It is noteworthy that the matrix $C(x)$ is assumed to be negatively definite in $D$, i.e., it does not have any zero eigenvalue. Moreover, $C(0)$ should be a normal matrix for the weighted Dirichlet problem to be well-posed. (If coefficients $B_{i}(x)$ have the main influence to the asymptotic of the solutions of system (1), then the last requirement is dispensable $[8,9])$. Therefore, it is important to consider the case where $C(0)$ has multiple zero eigenvalue and corresponding to it adjoint vectors.

Hence, the present article deals with a particular case of system (1) of the shape

$$
\Delta u-q(r) \Lambda u=0
$$

in the ball $\sum_{R}=\{x:|x|<R\} \subset R^{3}$ with the Dirichlet condition

$$
\left.u\right|_{S_{R}}=f .
$$

In this article, $\Lambda$ is a real constant non-negative definite $N \times N$ matrix having the eigenvalue $\lambda=0, q$ is scalar continuous function positive for $r \neq 0$ and such that

$$
q(r)=O\left(r^{-2 \alpha}\right), \alpha>1, \text { as } \quad r \rightarrow 0,
$$

$S_{R}=\partial \sum_{R}, f=\left(f_{1}, f_{2}, \ldots, f_{N}\right\}$ and $u=\left(u_{1}, u_{2}, \ldots, u_{N}\right)$ are the given and unknown vector functions, respectively. (Condition (5) means with respect to system (1) that $a(r)$ vanishes as $r \rightarrow 0$ not faster than any power of $r$.) Hence, the order of system (3) is strongly degenerate at the point $x=0$ because of $\alpha>1$.

Let $S$ be a non-degenerate matrix such that

$$
S \Lambda S^{-1}=J_{\Lambda}=\operatorname{diag}\left(L_{m_{0}}\left(\lambda_{0}\right) L_{m_{1}}\left(\lambda_{1}\right) \ldots L_{m_{p}}\left(\lambda_{p}\right)\right)
$$

is the canonical Jordan form of $\Lambda$ with $m_{i} \times m_{i}$ lower blocks

$$
L_{m_{i}}\left(\lambda_{i}\right)=\left(\begin{array}{ccccc}
\lambda_{i} & 0 & \ldots & 0 & 0 \\
1 & \lambda_{i} & \ldots & 0 & 0 \\
\ldots & \ldots & \ldots & \ldots & \ldots \\
0 & 0 & \ldots & \lambda_{i} & 0 \\
0 & 0 & \ldots & 1 & \lambda_{i}
\end{array}\right), \quad i=\overline{0, p}
$$

Multiplying both (3) and (4) from the left by $S$, we get the system

$$
\Delta v-q(r) J_{\Lambda} v=0
$$

and the Dirichlet condition

$$
\left.v\right|_{S_{R}}=g,
$$

where $v=S u$, and $g=S f$. Therefore, system (6) and Dirichlet condition (7) can be split into $p+1$ separate systems

$$
\Delta v^{i}-q(r) L_{m_{i}}\left(\lambda_{i}\right) v^{i}=0,
$$


and Dirichlet conditions

$$
\left.v^{i}\right|_{S_{R}}=g^{i}, i=\overline{0, p}
$$

which correspond to the blocks of Jordan matrix $J_{\Lambda}$, where both $v^{i}$ and $g^{i}$ are $m_{i^{-}}$ dimensional vector functions. If $\Lambda$ is a matrix of simple structure, then all $m_{i}=1$, i.e., (6) splits into $N$ separate equations, obviously.

Let $\lambda_{0}=0$ and, for convenience, only one eigen vector corresponds to this eigenvalue of $\Lambda$. Then, $\operatorname{Re} \lambda_{i}<0$ for the rest $i=\overline{1, p}$, since the matrix $\Lambda$ is non-negatively defined. As mentioned above, the solvability of a Dirichlet type problem under the condition $\operatorname{Re} \lambda_{i}<0$ is investigated in [6,7].

The main aim of this article is to give a well-posedness of the Dirichlet type problems to the system

$$
\Delta v^{0}-q(r) L_{m_{0}}(0) v^{0}=0,
$$

which is in accordance with eigenvalue $\lambda_{0}=0$ of $\Lambda$. In order to avoid the complicated notations, instead of (8), we consider the system

$$
\Delta v-q(r) L_{s}(0) v=0,
$$

where $v=\left(v_{1}, v_{2}, \ldots, v_{s}\right)$ and $L_{s}(0)$ is a $s \times s$ lower Jordan block with zero diagonal entries. It is easily seen that

$$
\left\{\begin{aligned}
\Delta v_{1} & =0, \\
\Delta v_{k+1} & =q(r) v_{k}, \quad k=\overline{1, s-1},
\end{aligned}\right.
$$

is the outspread form of (9).

Denote $\Sigma_{R}^{0}=\Sigma_{R} \backslash\{x=0\}$. Let $\|v\|$ be the Euclidian norm of a vector $v$. We propose the two following statements of the Dirichlet type problem to system (9).

Problem $D_{1}$. Find a solution $v=\left(v_{1}, v_{2}, \ldots, v_{s}\right) \in C^{2}\left(\Sigma_{R}^{0}\right) \cup C\left(\Sigma_{R}^{0} \cup S_{R}\right)$ of Equation 9 that satisfies Dirichlet condition

$$
\left.v\right|_{S_{R}}=g
$$

and relation

$$
r\|v(x)\|=o(1), \quad \text { as } \quad x \rightarrow 0
$$

Problem $D_{2}$. Find a solution $v \in C^{2}\left(\Sigma_{R}^{0}\right) \cup C\left(\Sigma_{R}^{0} \cup S_{R}\right)$ of Equation 9 , such that it satisfies Dirichlet condition (11) and is bounded in $\Sigma_{R^{*}}^{0}$

\section{The properties of particular solutions of Equation 8}

Let $H_{n}^{m}(x)$ be $m$ th the harmonic of a homogeneous harmonic polynomial of degree $n$, ${ }^{\text {a }}$ i.e., $H_{n}^{m}(\lambda x)=\lambda^{n} H_{n}^{m}(x)$ and $\Delta H_{n}^{m}(x)=0$. Then, $r^{-n} H_{n}^{m}(x)=H_{n}^{m}(\omega)$ (here $\omega=x / r$ ) is the $m$ th spherical harmonic of order $n$ continuous on the unit sphere $S_{1}$. Let $c_{n m}$ be any constant vector, and let $Q_{n}(r)$ be a matrix solution of ODEs system

$$
l_{n}(w)-q(r) L_{s}(0) w=0, \quad 0<r<R,
$$

where

$$
l_{n}=\frac{\mathrm{d}^{2}}{\mathrm{~d} r^{2}}+\frac{2(n+1)}{r} \frac{\mathrm{d}}{\mathrm{d} r}
$$


and $w$ is an unknown $s$-dimensional vector function. Then, the functions

$$
v_{n m}(x)=r^{n} H_{n}^{m}(\omega) Q_{n}(r) c_{n m}, n=0,1,2, \ldots, \quad m=0, \pm 1, \ldots, \pm n,
$$

represent the particular solutions of system (9).

We seek for a solution $Q_{n}(r)$ of system (12), which satisfies condition $Q(R)=E$, where $E$ is the unit matrix. To this end, on the set of functions $\psi$ bounded on the interval $(0, R)$, we consider the integral operator

$$
\begin{gathered}
K_{n}(\psi)(r)=\int_{0}^{R} K_{n}(r, t) q(t) \psi(t) \mathrm{d} t, \\
K_{n}(r, t)=-\frac{t^{2 n+2}}{2 n+1} \times\left\{\begin{array}{l}
r^{-2 n-1}-R^{-2 n-1}, 0<t \leq r, \\
t^{-2 n-1}-R^{-2 n-1}, r \leq t \leq R,
\end{array}\right.
\end{gathered}
$$

and its integer powers

$$
K_{n}^{\sigma}(\psi)(r)=K_{n}\left(K_{n}^{\sigma-1}(\psi)\right)(r),
$$

where by definition $K^{0}(\psi)(x) \equiv \psi(x)$. Obviously, according to this definition

$$
\begin{aligned}
K_{n}^{\sigma}(\psi)(r)= & -\frac{1}{2 n+1}\left(\left(r^{-2 n-1}-R^{-2 n-1}\right) \int_{0}^{r} t^{2 n+2} q(t) \mathrm{K}_{n}^{\sigma-1}(\psi)(t) \mathrm{d} t\right. \\
& \left.+\int_{r}^{R} t\left(1-\left(\frac{t}{R}\right)^{2 n+1}\right) q(t) K_{n}^{\sigma-1}(\psi)(t) \mathrm{d} t\right), \quad \sigma=1,2, \ldots
\end{aligned}
$$

Lemma 1. Let relation (5) hold. If $n>\sigma(\alpha-1)-\frac{1}{2}$, then

$$
\left.K_{n}^{\sigma}(\psi)(r) \leq \frac{M_{\sigma}}{n^{\sigma}} r^{2 \sigma(1-\alpha)}\right) \text { on }(0, R)
$$

where $M_{\sigma}$ is some constant independent of $n$, and $K_{n}^{\sigma}(\psi)(R)=0(\sigma=1,2, \ldots)$.

Proof. We prove relation (15) by induction.

Since $\psi(r)$ is bounded on $(0, R)$, inequality (15) holds for $\sigma=0$ with some constant $M_{0}$. It follows from relation (5) that $0<q(r) \leq M r^{-2 \alpha} \forall r \in(0, R)$, where $M$ is a positive constant. Then, $q(t)|\psi(t)| \leq M M_{0} t^{-2 \alpha} \forall t \in(0, R)$. Assuming that $n>\alpha-\frac{3}{2}$, we obtain that

$$
\begin{aligned}
\left|K_{n}(\psi)(r)\right| & \leq \frac{M}{2 n+1}\left(r^{-2 n-1} \int_{0}^{r} t^{2(n+1-\alpha)} \mathrm{d} t+\int_{r}^{R} t^{1-2 \alpha} \mathrm{d} t\right) \\
& \leq \frac{M}{2 n+1}\left(\frac{1}{2 n-2 \alpha+3}+\frac{1}{2(\alpha-1)}\right) r^{2(1-\alpha)}
\end{aligned}
$$

i.e., the estimate

$$
\left|K_{n}(\psi)(r)\right| \leq \frac{M_{1}}{n} r^{2(1-\alpha)}
$$

with some constant $M_{1}$ independent of $n$ holds. Thus, the validity of (15) is proved for $\sigma=1$. 
Let (15) be valid for $\sigma=k-1$ under the condition $n>(k-1)(\alpha-1)-\frac{1}{2}$. Then,

$$
t^{2 n+2} q(t)\left|K_{n}^{k-1}(\psi)(t)\right| \leq \frac{M M_{k-1}}{n^{k-1}} t^{2(n+k(1-\alpha))} \quad \forall t \in(0, R),
$$

i.e., the first integral in expression (14) converges, if $n>k(\alpha-1)-\frac{1}{2}$, and

$$
\begin{aligned}
\left|K_{n}^{k}(\psi)(r)\right| & \leq \frac{M M_{k-1}}{n^{k-1}(2 n+1)}\left(r^{-2 n-1} \int_{0}^{r} t^{2(n+k(1-\alpha))} \mathrm{d} t+\int_{r}^{R} t^{2 k(1-\alpha)-1} \mathrm{~d} t\right) \\
& \leq \frac{M M_{k-1}}{n^{k-1}(2 n+1)}\left(\frac{1}{2 n+2 k(1-\alpha)+1}+\frac{1}{2 k(\alpha-1)}\right) r^{2 k(1-\alpha)} .
\end{aligned}
$$

Therefore, there exists a constant $M_{k}$ such that (15) holds for $\sigma=k$ under the condition $n>k(\alpha-1)-\frac{1}{2}$.

If $n>\sigma(\alpha-1)-\frac{1}{2}$, then the first integral on the right-hand side of (14) converges as $r \in(0, R)$, and, evidently, $K_{n}^{\sigma}(\psi)(R)=0$.

It is easy to verify that

$$
l_{n}\left(K_{n}^{\sigma}(\psi)\right)=q(r) K_{n}^{\sigma-1}(\psi)(r), \quad \sigma=1,2, \ldots,
$$

under the conditions of Lemma 1.

Note that $w_{1} \equiv 1$ and $w_{2}=r^{-2 n-1}$ are linearly independent solutions of the differential equation $l_{n}(w)=0$. Thus, if $w$ is the solution of this equation such that $w(r)=o\left(r^{-2 n-1}\right)$ as $r \rightarrow 0$, then $w(r) \equiv$ const.

Denoting, as usual, by $[a]$ the integer part of the real number $a$, we introduce the integer $\alpha_{k}=\left[k(\alpha-1)+\frac{1}{2}\right]$, where $k$ is a non-negative integer. (Note that $\alpha_{0}=0$.)

We use below denotation $K_{n}^{\sigma-1}(r)=K_{n}^{\sigma-1}(\psi)(r)$ in the case $\psi(x) \equiv 1$.

Theorem 1. Let relation (5) hold. If $n \geq \alpha_{s-1}$, then there exists a unique matrix solution $Q_{n}(r)=\left\{q_{n i j}(r)\right\}$ of Equation 12 such that

$$
q_{n i j}(r)=o\left(r^{-2 n-1}\right), \text { as } \quad r \rightarrow 0, \quad i, j=\overline{1, s},
$$

and

$$
Q_{n}(R)=E .
$$

Proof. Let the condition $n \geq \alpha_{s-1}$ be valid. Then, according to Lemma 1, the functions $\sigma=\overline{0, s-1}, \sigma=\overline{0, s-1}$, are continuous on the interval $(0, R)$. Introduce the $s \times s$ matrix $Q_{n}(r)=\left\{q_{n i j}(r)\right\}$ by the formula

$$
q_{n i j}(r)=\left\{\begin{array}{cl}
0, & \text { if } i<j, \\
K_{n}^{i-j}(r), & \text { if } i \geq j .
\end{array}\right.
$$

Note that estimate (15) yields the relations

$$
q_{n i j}(r)=O\left(r^{2(i-j)(1-\alpha)}\right), \text { as } \quad r \rightarrow 0, \quad 1 \leq j \leq i \leq s .
$$


This implies the validity of condition (17), because $2 n+1>2(s-1)(\alpha-1) \geq 2(i-j)(\alpha-$ $1)$, for $i, j=\overline{1, s}$. Moreover,

$$
l_{n}\left(Q_{n}\right)=q(r) L_{s}(0) Q_{n} \text { on }(0, R)
$$

because of (16), i.e., $Q_{n}$ is the matrix solution of Equation 12. Evidently, equality (18) follows from (14).

It remains to prove the uniqueness of the solution of problems (12), (17), and (18). Let $\tilde{Q}_{n}(r)=\left\{\tilde{q}_{n i j}\right\}$ be a matrix solution of system (12) continuous on $(0, R)$, and satisfying both conditions $\tilde{q}_{n i j}(r)=o\left(r^{-2 n-1}\right)$, as $r \rightarrow 0$, and $\tilde{Q}_{n}(R)=\Theta$, where $\Theta$ is zero matrix. Then, the equalities

$$
\begin{aligned}
l_{n}\left(\tilde{q}_{n 1 j}\right) & =0, \\
l_{n}\left(\tilde{q}_{n, k+1, j}\right) & =q(r) \tilde{q}_{n k j}(r), \quad k=\overline{1, s-1,}
\end{aligned}
$$

on the interval $(0, R)$ hold. Since $\tilde{q}_{n 1 j}(r)=o\left(r^{-2 n-1}\right)$ as $r \rightarrow 0$, we obtain that $\tilde{q}_{n 1 j}(r)=$ const on the interval $(0, R)$. Then, the condition $\tilde{q}_{n 1 j}(R)=0$ yields the identity $\tilde{q}_{n 1 j}(r) \equiv 0$ because of the continuity of the function $\tilde{q}_{n 1 j}$ on $(0, R)$. In such a case, the elements of the second row of matrix $\tilde{Q}_{n}$ satisfy the equation $l_{n}\left(\tilde{q}_{n 2 j}\right)=0$. For the same reason as above, we obtain that $\tilde{q}_{n 2 j}(r) \equiv 0(j=\overline{1, s})$ on $(0, R)$. Further, continuing this process, we get that $\tilde{q}_{n 3 j}(r) \equiv 0, \ldots, \tilde{q}_{n s j}(r) \equiv 0(j=\overline{1, s})$ on $(0, R)$. Hence, $\tilde{Q}_{n}(r) \equiv \Theta$ on $(0, R)$. This yields the uniqueness of the solution of problems (12), (17), and (18).

What is the structure of the solutions of system (12) that increase slower than $r^{-2 n-1}$ in the case where $n$ does not satisfy the condition $n \geq \alpha_{s-1}$ ? In order to get the answer to this question, we introduce $s \times s$ matrices $E_{k}=\left\{e_{i j}^{(k)}\right\}(k=\overline{1, s})$ with entries $e_{i i}^{(k)}=1$ for $s-k+1 \leq i \leq s$, and $e_{i j}^{(k)}=0$ for all rest $i$ and $j$. (Note that $E_{s}=E$ according to this definition.) Let us compose the matrixes

$$
Q_{n}^{(k)}(r)=Q_{n}(r) E_{k}, \quad k=\overline{1, s},
$$

where $Q_{n}$ is matrix elements of which are given by (19). It is easily seen that $Q_{n}^{(s)}(r)=Q_{n}(r) E=Q_{n}(r)$, and the elements $q_{n i j}^{(k)}(r)$ of rest matrixes $Q_{n}^{(k)}(r)(k=\overline{1, s-1})$ are defined by following formula

$$
q_{n i j}^{(k)}(r)=\left\{\begin{array}{c}
K_{n}^{i-j}(r), \quad \text { if } s-k+1 \leq j \leq i \leq s, \\
0, \quad \text { if } 1 \leq j \leq s-k \text { and } i>j .
\end{array}\right.
$$

If $n \geq \alpha_{k-1}$, then the powers $\mathrm{K}_{n}^{i-j}(r)$ exist for all $i$ and $j$ such that $s-k+1 \leq j \leq i \leq s$, and according to (20), the relation

$$
q_{n i j}^{(k)}(r)=O\left(r^{2(i-j)(1-\alpha)}\right)=o\left(r^{-2 n-1}\right), \text { as } \quad r \rightarrow 0
$$

holds. Moreover, we obtain by direct calculation that

$$
\begin{gathered}
l_{n}\left(Q_{n}^{(k)}\right)=q(r) L_{s}(0) Q_{n}^{(k)} \text { on }(0, R), \\
Q_{n}^{(k)}(R)=E_{k}
\end{gathered}
$$

for $\forall k=\overline{1, s-1}$ due to the definition of matrix $Q_{n}^{(k)}$. Hence, there holds the following 
Theorem 2. Let relation (5) hold, and let natural $k, 1 \leq k \leq s-1$, be such that $\alpha_{k+1} \leq$ $n \alpha_{k}$. Then, there exists a unique matrix solution $Q_{n}^{(k)}(r)=\left\{q_{n i j}^{(k)}(r)\right\}$ of Equation 12 such that relation (21) holds, and boundary value condition (22) is satisfied.

The uniqueness of the matrix solution $Q_{n}^{(k)}$ can be proved in the same way as that of the matrix solution $Q_{n}$. In this case, condition (21) is essential, just similar to condition (17) in Theorem 1.

Hence, we obtain to system (9) the following set of particular solutions (see (13)):

$$
\begin{gathered}
v_{n m}^{(k)}=\left(\frac{r}{R}\right)^{n} H_{n}^{m}(\omega) Q_{n}^{(k)}(r) c_{n m} \text { for } n_{k-1} \leq n<\alpha_{k}, \quad k=\overline{1, s-1}, \\
v_{n m}^{(s)}=\left(\frac{r}{R}\right)^{n} H_{n}^{m}(\omega) Q_{n}(r) c_{n m} \text { for } n \geq \alpha_{s-1},
\end{gathered}
$$

where $c_{n m}$ is arbitrary constant column vector.

\section{Existence and uniqueness of the solutions of problems $D_{1}$ and $D_{2}$}

Let us compose the superposition

$$
\begin{gathered}
v=\sum_{k=1}^{s-1} \sum_{\alpha_{k-1} \leq n<\alpha_{k}}\left(\frac{r}{R}\right)^{n} Q_{n}^{(k)}(r) \sum_{|m| \leq n} H_{n}^{m}(\omega) c_{n m} \\
+\sum_{n=\alpha_{s-1}}^{\infty}\left(\frac{r}{R}\right)^{n} Q_{n}(r) \sum_{|m| \leq n} H_{n}^{m}(\omega) c_{n m}
\end{gathered}
$$

of the particular solutions obtained above. Note, if $\alpha_{k_{0}}=0$ for some $k_{0}, 1 \leq k_{0} \leq s-1$, then $\alpha_{k}=0$ for all natural $k \leq k_{0}-1$. (Such a situation can come to exist, if $\alpha<2$.) Therefore, all the sums $\sum_{\alpha_{k-1} \leq n<\alpha_{k}}$ in (23), in which the inequality $\alpha_{k-1}<\alpha_{k}$ is impossible, are taken to be equal to zero.

Evidently, if the series (23) converges and its sum $v$ is twice differentiable in the spherical layer $\Sigma_{R}^{\delta}=\{x: \delta<|x|<R\}$ with arbitrarily small $\delta$, then this series satisfies system (9) in the ball $\Sigma_{R^{*}}^{0}$. Note that

$$
\begin{aligned}
\left.v\right|_{S_{R}}= & \sum_{k=1}^{s-1} \sum_{\alpha_{k-1} \leq n<\alpha_{k}} \sum_{|m| \leq n} H_{n}^{m}\left(\frac{x}{R}\right) E_{k} c_{n m} \\
& +\sum_{n=\alpha_{s-1}}^{\infty} \sum_{|m| \leq n} H_{n}^{m}\left(\frac{x}{R}\right) c_{n m}
\end{aligned}
$$

due to both (18) and (22).

Assume that the boundary vector function $g=\left(g_{1}, g_{2}, \ldots, g_{s}\right)$ (see (10)) is twice differentiable on unit sphere $S_{1}$. Thus, it can be expressed on the sphere $S_{R}$ by Laplace series [10]:

$$
g(x)=\sum_{n=0}^{\infty} \sum_{|m| \leq n} H_{n}^{m}(\omega) a_{n m}, \quad x \in S_{R}
$$

which converge (component-wise) uniformly and absolutely according to the assumed smoothness of the vector function $g$. The coefficients $a_{n m}=\left(a_{n m}^{(1)}, a_{n m}^{(2)}, \ldots, a_{n m}^{(s)}\right)$ in (25) can be calculated as follows ${ }^{b}$ :

$$
a_{n m}^{(i)}=\frac{2 n+1}{4 \pi R^{2}} \frac{(n-m) !}{(n+m) !} \int_{S_{R}} h_{i}(\varphi, \vartheta) Y_{n}^{m}(\varphi, \vartheta) \mathrm{d} \varphi \mathrm{d} \vartheta, \quad|m| \leq n
$$


where $h_{i}(\phi, \vartheta)=g_{i}(x)$, for $|x|=R$ and $Y_{n}^{m}(\varphi, \vartheta)=H_{n}^{m}(\omega), \phi$, $\vartheta(0 \leq \phi \leq 2 \pi, 0 \leq \vartheta \leq$ $\pi)$ are spherical coordinates which are introduced by the rule: $x_{1}=r \sin 9 \cos \phi, x_{2}=r$ $\sin 9 \sin \phi$, and $x_{3}=r \cos 9$.

It is easily seen that series (24) coincides with series (25), if $c_{n m}=a_{n m}$ for $n \geq \alpha_{s-1}$, and $E_{k} c_{n m}=a_{n m}$ for $\alpha_{k-1} \leq n<\alpha_{k}, k=\overline{1, s-1}$, i.e., if components $h_{1}, h_{2}, \ldots, h_{s-1}$ of vector function $h$ satisfy the following orthogonality conditions

$$
\iint_{S_{R}} h_{k}(\varphi, \vartheta) Y_{n}^{m}(\varphi, \vartheta) \mathrm{d} \varphi \mathrm{d} \vartheta=0 \text { for } 0 \leq n<\alpha_{s-k}, \quad k=\overline{1, s-1},
$$

on sphere $S_{R}$. Let us consider series (23), in which $c_{n m}=a_{n m}$ :

$$
\begin{gathered}
v=\sum_{k=1}^{s-1} \sum_{\alpha_{k-1} \leq n<\alpha_{k}}\left(\frac{r}{R}\right)^{n} Q_{n}^{(k)}(r) \sum_{|m| \leq n} H_{n}^{m}(\omega) a_{n m}+ \\
\sum_{n=\alpha_{s-1}}^{\infty}\left(\frac{r}{R}\right)^{n} Q_{n}(r) \sum_{|m| \leq n} H_{n}^{m}(\omega) a_{n m} .
\end{gathered}
$$

Assume that condition (26) is fulfilled in addition to the smoothness of $g$. Then, $\left.v\right|_{S_{R}}=g$, i.e., series (27) converges (component-wise) uniformly and absolutely on the sphere $S_{R}$.

We shall prove that series (27) converges uniformly and absolutely in the spherical layer $\Sigma_{R}^{\delta}$ with arbitrarily small $\delta$. Note that components $v_{i}(i=\overline{1, s})$ of the vector function $v=\left(v_{1}, v_{2}, \ldots, v_{s}\right)$ in (27) can be formally represented in the form

$$
v_{1}=w_{1}(x), \quad v_{k}=w_{k}(x)+W_{k}(x), \quad k=\overline{2, s},
$$

where

$$
\begin{aligned}
w_{k}(x) & =\sum_{n=\alpha_{s-k}}^{\infty}\left(\frac{r}{R}\right)^{n} \sum_{|m| \leq n} a_{n m}^{(k)} H_{n}^{m}(\omega), \quad k=\overline{1, s}, \\
W_{k}(x) & =\sum_{l=1}^{k-1} \sum_{n=\alpha_{s-l}}^{\infty}\left(\frac{r}{R}\right)^{n} \mathrm{~K}_{n}^{k-l}(r) \sum_{|m| \leq n} a_{n m}^{(l)} H_{n}^{m}(\omega), \quad k=\overline{2, s} .
\end{aligned}
$$

The terms

$$
\left(\frac{r}{R}\right)^{n} \sum_{|m| \leq n} a_{n m}^{(k)} H_{n}^{m}(\omega)
$$

of the series on the right-hand side of (29) are harmonic functions in $\Sigma_{R}$. Since these series converge uniformly on the sphere $S_{R}$, they also converge uniformly in $\Sigma_{R}$, and their sums $w_{k}(r, \omega), k=\overline{1, s}$, are harmonic functions in $\Sigma_{R}$ because of Harnack's theorem [11].

Further, according to Lemma 1 estimates

$$
K_{n}^{k-l}(r) \leq \frac{M_{k-l}}{n^{k-l}} r^{2(k-l)(1-\alpha)}, \quad l=\overline{k-1, s+k-1,}
$$

hold, where $n \geq \alpha_{k-l}$ and $M_{k-l}$ is a constant independent of $n$. Consequently,

$$
\left|\left(\frac{r}{R}\right)^{n} K_{n}^{k-l}(r) \sum_{|m| \leq n} H_{n}^{m}(\omega) a_{n m}^{(l)}\right|<\frac{M_{k-l}}{n^{k-l}} r^{2(k-l)(1-\alpha)} \sum_{|m| \leq n}\left|H_{n}^{m}(\omega) a_{n m}^{(l)}\right|
$$


in $\Sigma_{R}^{\delta}$ for $\forall n \geq \alpha_{k-l}$. Note that the constants $M_{k}, k=\overline{1, s-1}$, do not depend on $n$ as well as on $\delta$. Evidently, they yield the uniform and absolute convergence of series (30) in $\overline{\Sigma_{R}^{\delta}}=\Sigma_{R}^{\delta} \cup S_{R} \cup S_{\delta}$.

Let $\mathrm{G}_{\delta}(x, \xi)$ be the Green function of the Dirichlet problem to Laplace equation in $\Sigma_{R}^{\delta}$, and let $w_{k n}(x)$ and $W_{k n}(x)$ be the $n$th partial sum of corresponding series (29) and (30). Since

$$
\Delta W_{2 n}(x) \equiv q(r) w_{1 n}(x),
$$

in $\Sigma_{R}^{0}$, relations

$$
\begin{gathered}
W_{2 n}(x)=\int_{\Sigma_{R}^{\delta}} G_{\delta}(x, \xi) w_{1 n}(\xi) \mathrm{d} \sigma_{\xi}, \\
W_{k n}(x)=\int_{\Sigma_{R}^{\delta}} G_{\delta}(x, \xi)\left(w_{(k-1) n}(\xi)+W_{(k-1) n}(\xi)\right) \mathrm{d} \sigma_{\xi}, \quad k=\overline{3, s},
\end{gathered}
$$

hold, where $\mathrm{d} \sigma_{\xi}$ is a volume element of $\Sigma_{R^{\prime}}^{\delta}$ These yield the equalities

$$
\begin{aligned}
& \frac{\partial^{2} W_{2 n}(x)}{\partial x_{i}^{2}}=\int_{\Sigma_{R}^{\delta}} \frac{\partial^{2} G_{\delta}(x, \xi)}{\partial x_{i}^{2}} w_{1 n}(\xi) \mathrm{d} \sigma_{\xi}, \\
& \frac{\partial^{2} W_{k n}(x)}{\partial x_{i}^{2}}=\int_{\Sigma_{R}^{\delta}} \frac{\partial^{2} G_{\delta}(x, \xi)}{\partial x_{i}^{2}}\left(w_{(k-1) n}(\xi)+W_{(k-1) n}(\xi)\right) \mathrm{d} \sigma_{\xi}, \quad k=\overline{3, s} .
\end{aligned}
$$

Owing to the uniform and absolute convergence in $\overline{\Sigma_{R}^{\delta}}$ of sequences $\left\{w_{k n}(r, \omega)\right\}$ and $\left\{W_{k n}(r, \omega)\right\}$, as $n \rightarrow \infty$, we obtain, from (31) and (32), coherently, that the functions $w_{k}(r, \omega)$ and $W_{k}(r, \omega)$, defined by (29) and (30), are twice differentiable and

$$
\begin{gathered}
\frac{\partial^{2} w_{k}(x)}{\partial x_{i}^{2}}=\sum_{n=\alpha_{s-k}}^{\infty} \frac{\partial^{2}}{\partial x_{i}^{2}}\left(\left(\frac{r}{R}\right)^{n} \sum_{|m| \leq n} a_{n m}^{(1)} H_{n}^{m}(\omega)\right), \quad k=\overline{1, s}, \\
\frac{\partial^{2} W_{k}(x)}{\partial x_{i}^{2}}=\sum_{l=1}^{k-1} \sum_{n=\alpha_{s-l}}^{\infty} \frac{\partial^{2}}{\partial x_{i}^{2}}\left(\left(\frac{r}{R}\right)^{n} K_{n}^{k-l}(r) \sum_{|m| \leq n} a_{n m}^{(l)} H_{n}^{m}(\omega)\right), \quad k=\overline{2, s},
\end{gathered}
$$

in $\Sigma_{R}^{\delta}(i=1,2$, and 3$)$.

Hence, the vector function $v=\left(v_{1}, v_{2}, \ldots, v_{s}\right)$ with the components $v_{i}$ defined by (28)(30) is from class $C^{2}\left(\Sigma_{R}^{0} \cup S_{R}\right)$, and it satisfies system (9) in $\Sigma_{R}^{0}$ and the Dirichlet condition $\left.v\right|_{S_{R}}=g$, only if orthogonality conditions (26) hold. Besides, it follows from Lemma 1 that

$$
v_{k}(x)=O\left(r^{\alpha_{s-1}-2(k-1)(\alpha-1)}\right), \text { as } x \rightarrow 0, \quad k=\overline{1, s} .
$$

Therefore, $r\|v(x)\|=o(1)$ as $x \rightarrow 0$, if

$$
\alpha_{s-1}-2(s-1)(\alpha-1)>-1 .
$$

Note that this inequality holds, if, for instance,

$$
1<\alpha<\frac{2 s-1}{2(s-1)}
$$


We prove thereby the existence of the solution of problem $D_{1}$, if both $\alpha$ and $s$ are related by (33).

If the coefficients $a_{n m}^{(k)}(k=\overline{1, s-1})$ in (28) and (29) are such that

$$
a_{n m}^{(k)}=0 \text { for } 0 \leq n<2(s-k)(\alpha-1),
$$

i.e., the components $h_{k}(k=\overline{1, s-1})$ of the vector function $h$ satisfy the orthogonality conditions

$$
\iint_{S_{R}} h_{k}(\varphi, \vartheta) Y_{n}^{m}(\varphi, \vartheta) \mathrm{d} \varphi \mathrm{d} \vartheta=0 \text { for } 0 \leq n<2(s-k)(\alpha-1),
$$

then the solution $v$ of system (8), given by (28)-(30), is bounded in $\Sigma_{R}^{0}$ and continuous in $\bar{\Sigma}_{R}$. Thus, under ortogonality conditions (34), we obtain the solution $v=\left(v_{1}, v_{2}\right.$, ..., $v_{s}$ ) of problem $D_{2}$ of the shape

$$
v_{k}(x)=\sum_{l=1}^{k} \sum_{n=n_{s-l}}^{\infty}\left(\frac{r}{R}\right)^{n} K_{n}^{k-l}(r) \sum_{|m| \leq n} a_{n m}^{(l)} H_{n}^{m}(\omega), \quad k=\overline{1, s}
$$

where

$$
n_{k}=\left\{\begin{array}{l}
2 k(\alpha-1), \quad \text { if } 2 k(\alpha-1) \text { is an integer } \\
{[2 k(\alpha-1)]+1 \quad \text { in the opposite case. }}
\end{array}\right.
$$

The uniqueness of the solutions of both the problems $D_{1}$ and $D_{2}$ yields the following lemma.

Lemma 2. Let $v=\left(v_{1}, v_{2}, \ldots, v_{s}\right)$ be a solution of problem $D_{1}$ or problem $D_{2}$ with the homogeneous Dirichlet condition $\left.v\right|_{S_{R}}=0$. If relation (33) holds, then $v_{i}=0$ in $\Sigma_{R}^{0}(i=\overline{1, n})$.

Proof. Assume that $v=\left(v_{1}, v_{2}, \ldots, v_{s}\right)$ is a solution of problem $D_{1}$. Since $\Delta v_{1}=0$ in $\Sigma_{R}^{0}$ and $\left.v_{1}\right|_{S_{R}}=0$, we get that $v_{1} \equiv 0$ in $\Sigma_{R}^{0}$ because of the relation $v_{1}(x)=o\left(r^{-1}\right)$, as $x \rightarrow 0$, which holds because of the validity of condition (11). Then, it follows from system (9) that $\Delta v_{2}=0$ in $\Sigma_{R}^{0}$. Both the conditions $\left.v_{2}\right|_{S_{R}}=0$ and $v_{2}(x)=o\left(r^{-1}\right)$, as $x \rightarrow 0$, yield the identity $v_{2} \equiv 0$ in $\Sigma_{R}^{0}$ to (11). Continuing this process, we obtain that all the components $v_{i} \equiv 0(i=\overline{1, n})$ in $\Sigma_{R^{*}}^{0}$

If $v=\left(v_{1}, v_{2}, \ldots, v_{s}\right)$ is a solution of problem $D_{2}$, then it satisfies (11), too. This implies the identity $v \equiv 0$ in $\Sigma_{R}^{0}$, without doubt.

One can summarize the reasoning given above as follows:

Theorem 3. Let $g \in C^{2}\left(S_{R}\right)$, and let relation (5) hold. If orthogonality conditions (26) are fulfilled, and the parameters $\alpha$ and $s$ satisfy inequality (33), then there exists a unique solution $v$ of problem $D_{1}$, which can be represented by formulas (28)-(30). If orthogonality conditions (34) hold, then there exists a unique solution $v$ of problem $D_{2}$ with the components $v_{i}$ of the shape (35).

\section{Endnotes}

a One can express the spherical function $H_{n}^{m}(x)$ in Cartesian coordinates $x=\left(x_{1}, x_{2}, x_{3}\right)$ by formula [12]:

$$
H_{n}^{m}(x)=r^{n}\left(r^{2}-x_{3}^{2}\right)^{-\frac{m}{2}} P_{n}^{|m|}\left(x_{3} / r\right) \times\left\{\begin{array}{c}
\operatorname{Re}\left(x_{1}-i x_{2}\right), \quad \text { if } 0 \leq m \leq n, \\
\operatorname{Im}\left(x_{1}-i x_{2}\right), \quad \text { if }-n \leq m<0,
\end{array}\right.
$$


where $P_{n}^{m}$ is adjoint Legendre's function, and $i$ is the imaginary unit. ${ }^{b}$ Our opinion is that spherical coordinates are more convenient than Cartesian in the calculation of the coefficients $a_{n m}$ of series (25). The matter is such that spherical functions $Y_{n}^{m}(\varphi, \vartheta)$ have quite a simple expresion:

$$
Y_{n}^{m}(\varphi, \vartheta)=P_{n}^{m}(\cos \vartheta) \times\left\{\begin{array}{c}
\cos m \varphi,-n \leq m \leq 0 \\
\sin m \varphi, 0<m \leq n
\end{array}\right.
$$

Received: 11 April 2011 Accepted: 22 August 2011 Published: 22 August 2011

\section{References}

1. Mikhailov, LG: Elliptic equations with singular coefficients. Izv Akad Nauk SSSR, Ser Math 26, 293-312 (1962). (Russian)

2. Achildiyev, SA: First and second boundary value problems for elliptic equations degenerating at the inner points of finite number. Dokl Akad Nauk SSSR 152(1), 13-16 (1963). (Russian)

3. Yanushauskas, A: On the elliptic equations coefficients of which have high order singularities under the lower derivatives. Sibirsk Math Zh 17(5), 1176-1187 (1976). (Russian)

4. Baldes, A: Degenerate elliptic equations, diagonal systems and variational integrals. Manuscripta Math. 55, 467-486 (1986). doi:10.1007/BF01186659

5. Rutkauskas, S: On the first boundary value problem for the class of elliptic systems degenerating at an inner point. Math Model Anal. 6(1), 147-155 (2001)

6. Rutkauskas, S: On the Dirichlet problem for a system of degenerate at a point elliptic equations in the class of bounded functions. Lithuanian Math J. 44(1), 85-104 (2004). doi:10.1023/B:LIMA.0000019860.48310.6b

7. Rutkauskas, S: On the Dirichlet problem with an asymptotic condition for an elliptic system strongly degenerate at a point. Lithuanian Math J. 41(4), 384-393 (2001). doi:10.1023/A:1013816706017

8. Rutkauskas, S: The Dirichlet problem with asymptotic conditions for an elliptic system degenerate at a point. I Differ Equ. 38(3), 405-412 (2002). doi:10.1023/A:1016070111630

9. Rutkauskas, S: The Dirichlet problem with asymptotic conditions for an elliptic system degenerate at a point. II Differ Equ. 38(5), 719-725 (2002). doi:10.1023/A:1020223027249

10. Koschmieder, L: Monatsh Math Phys. 40, 223-232 (1933). doi:10.1007/BF01708866

11. Gilbarg, D, Trudinger, N: Elliptic Partial Differential Equations of Second Order. Springer, Berlin (1983)

12. Bateman, H, Erdélyi, A: Higher Transcendental Functions, V. 2. MC Graw-Hill, New York (1953)

doi:10.1186/1687-2770-2011-16

Cite this article as: Rutkauskas: On the solvability conditions of the first boundary value problem for a system of elliptic equations that strongly degenerate at a point. Boundary Value Problems 2011 2011:16.

\section{Submit your manuscript to a SpringerOpen ${ }^{\odot}$ journal and benefit from:}

- Convenient online submission

- Rigorous peer review

- Immediate publication on acceptance

- Open access: articles freely available online

- High visibility within the field

- Retaining the copyright to your article

Submit your next manuscript at $\mathbf{s p r i n g e r o p e n . c o m ~}$ 